\title{
Cartilage-dependent Subchondral Ossification in Rheumatoid Arthritis
}

RIKAKO HIRAMATSU, MD, Nephrology Center, Toranomon Hospital; YOSHIFUMI UBARA, MD, Nephrology Center and Okinaka Memorial Institute for Medical Research, Toranomon Hospital; TATSUYA SUWABE, MD; JUNICHI HOSHINO, MD; KEIICHI SUMIDA, MD; EIKO HASEGAWA, MD; MASAYUKI YAMANOUCHI, MD; NORIKO HAYAMI, MD; NAOKI SAWA, MD, Nephrology Center, Toranomon Hospital; KENMEI TAKAICHI, MD, Nephrology Center and Okinaka Memorial Institute for Medical Research, Toranomon Hospital, Tokyo, Japan. Address correspondence to Dr. Y. Ubara, Nephrology Center, Toranomon Hospital, 2-2-2 Toranomon, Minato-ku, Tokyo, Japan 105-8470. E-mail: ubara@toranomon.gr.jp. J Rheumatol

2013;40:1923-4; doi:10.3899/jrheum.130201

We observed that, in rheumatoid arthritis (RA), subchondral bone may have a lamellar structure arranged in a vertical direction because of the cushioning effect of cartilage, while bone at the sites of cartilage defects develops a woven structure due to the lack of a cushioning effect.

A 58-year-old Japanese woman with a 15-year history of RA was admitted to our hospital for surgery on the left knee. Her RA had been treated using various antirheumatic drugs, including gold derivatives, penicillamine, methotrexate, steroids, and a soluble tumor necrosis factor receptor inhibitor (etanercept), but these medications had not been effective. She had also undergone operations including plate fixation of the cervical spine, left hip replacement, and right knee replacement. C-reactive protein (CRP) was $6.8 \mathrm{mg} / \mathrm{dl}$, rheumatoid factor $33 \mathrm{U} / \mathrm{ml}$ (normal $<10 \mathrm{U} / \mathrm{ml}$ ), anticyclic citrullinated peptide antibody $47 \mathrm{U} / \mathrm{ml}$ (normal $<4.5 \mathrm{U} / \mathrm{ml}$ ), and matrix metalloproteinase- 3 was $421 \mathrm{ng} / \mathrm{ml}$ (normal $17-60 \mathrm{ng} / \mathrm{ml}$ ). The Disease Activity Score-CRP was calculated to be 8.3 .

Radiography showed narrowing of the left knee joint
(Figure 1A), and there was extensive sclerosis on a coronal view on computed tomography (Figure 1B). A biopsy specimen was obtained from the proximal epiphysis of the tibia for histomorphometric evaluation after Villanueva staining of undecalcified sections. Sclerosis of the tibia was seen at the region denuded of cartilage, while the region with cartilage cover was nonsclerotic. In the former region, thickened cortical bone covered cancellous bone (Figure 2) that had a woven structure with irregularly arranged trabeculae (Figure 3A). In the latter region, the subchondral bone consisted of thin cortex and cancellous bone (Figure 2) with lamellar trabeculae growing in a vertical direction (Figure 3B).

Cartilage provides a cushioning effect for load-bearing joints and helps maintain the integrity of subchondral bone. In patients with RA, the articular cartilage is resorbed through the action of collagenase released from the synovial pannus, resulting in loss or narrowing of the joint space. The subchondral bone is also eventually affected. The findings in our patient suggested that subchondral bone has a

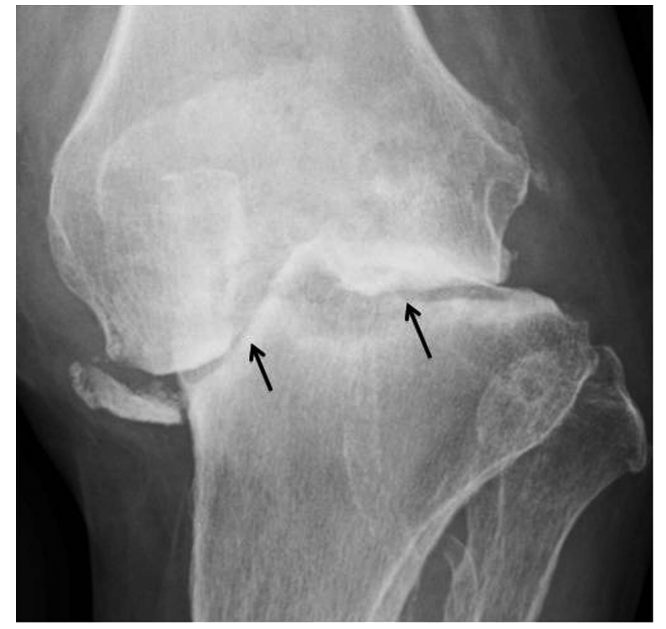

(a)

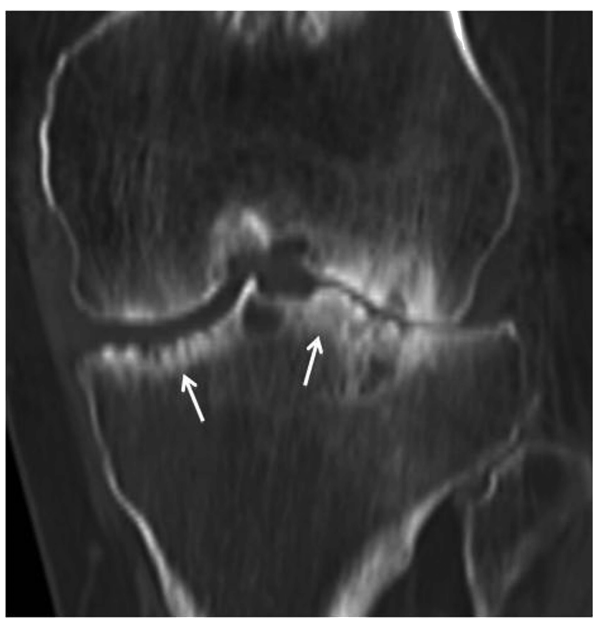

(b)

Figure 1. A. Radiograph shows narrowing of the left knee joint. B. Computed tomography scan reveals extensive sclerosis in a coronal view. 
lamellar structure arranged in a vertical direction by the cushioning effect of cartilage, while bone at the sites of cartilage defects develops a woven structure because of the lack of a cushioning effect.

\section{ACKNOWLEDGMENT}

We thank Akemi Ito of the Ito Bone Histomorphometry Institute for performing histomorphometric analysis.

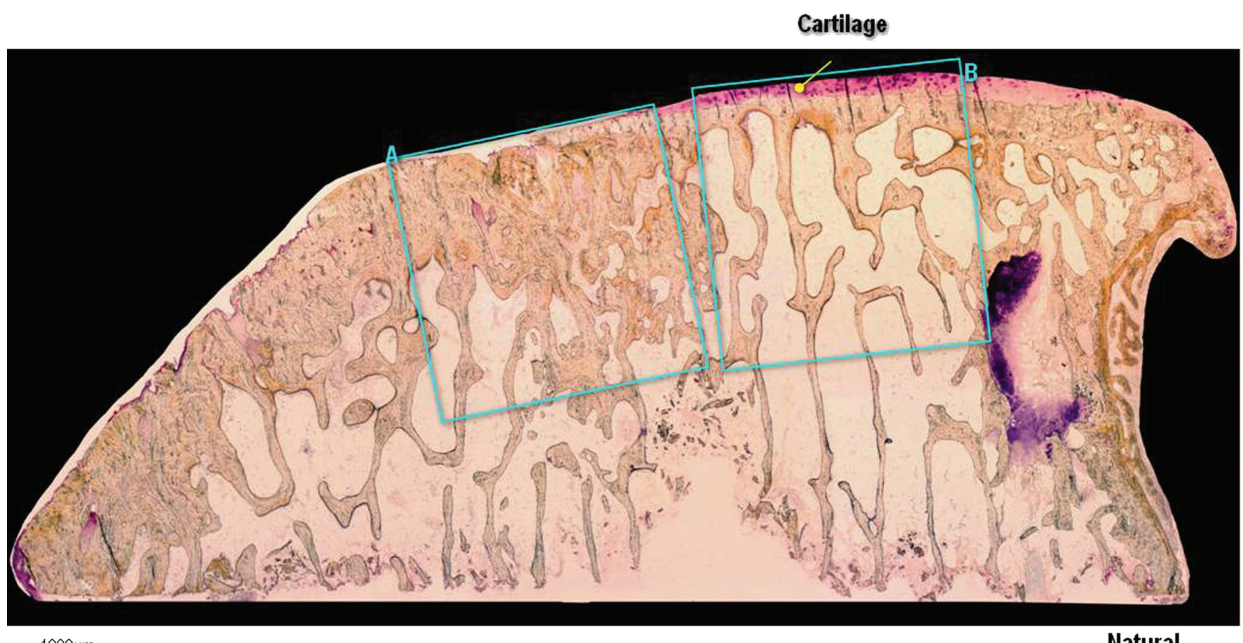

$1000 \mu \mathrm{m}$

Natural

Figure 2. A biopsy specimen from the proximal epiphysis of the tibia shows sclerosis at the region denuded of cartilage, while the region with cartilage cover was nonsclerotic (Villanueva stain; original magnification $\times 25$ ).

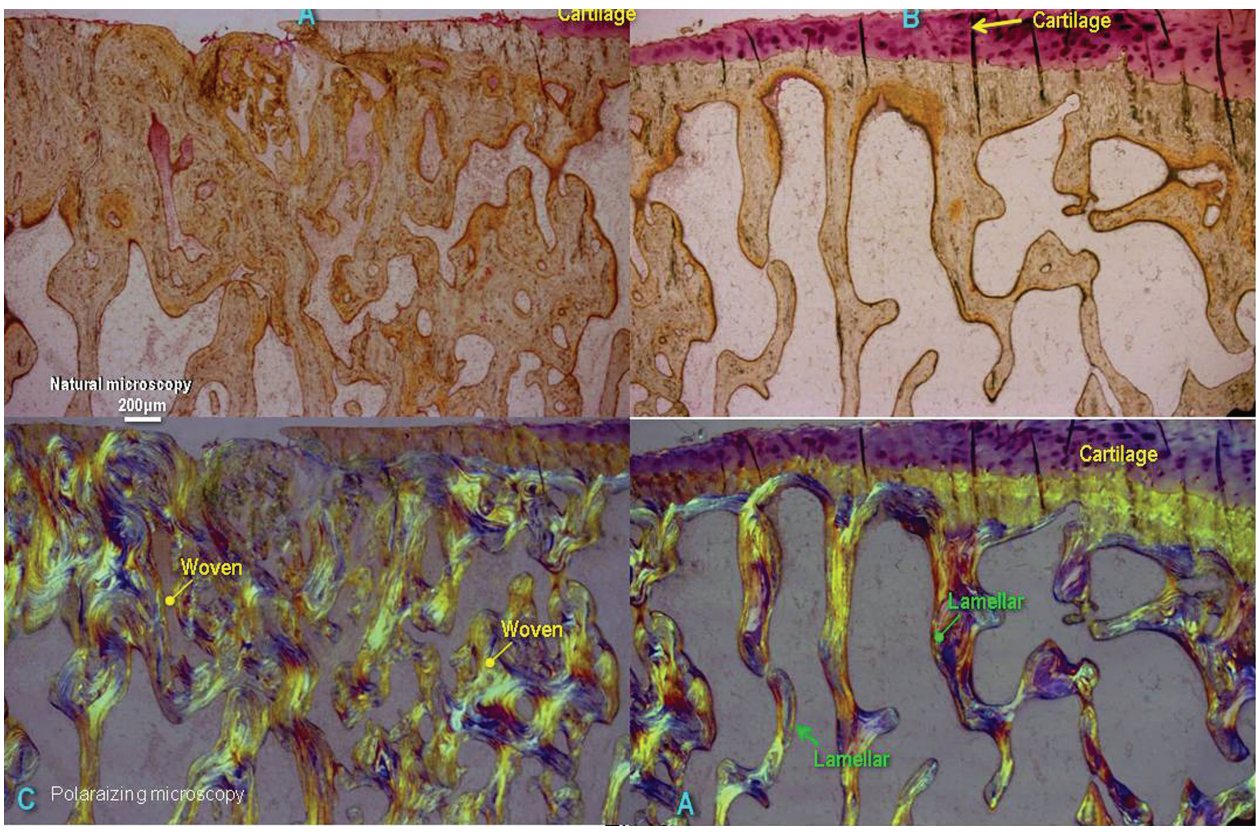

Figure 3. A. Thickened cortical bone covers cancellous bone that has a woven structure with irregularly arranged trabeculae. B. Subchondral bone consisted of thin cortex and cancellous bone with lamellar trabeculae growing in a vertical direction (original magnification $\times 40$ ). 